\title{
Perfect fluid cosmologies with varying light speed
}

\author{
Luis P. Chimento and Alejandro S. Jakubi \\ Departamento de Física, \\ Facultad de Ciencias Exactas y Naturales, \\ Universidad de Buenos Aires \\ Ciudad Universitaria, Pabellón I, \\ 1428 Buenos Aires, Argentina.
}

May 1, 2002

\begin{abstract}
We have found exact constant solutions for the cosmological density parameter using a generalization of general relativity that incorporates a cosmic time-variation of the velocity of light in vacuum and the Newtonian gravitation constant. We have determined the conditions when these solutions are attractors for an expanding universe and solved the problems of the Standard Big Bang model for perfect fluids.
\end{abstract}

PACS Numbers: 98.80.Cq, 98.80.-k, 95.30.Sf 


\section{Introduction}

Recent observations suggest a universe that is lightweight: matter density about one-third the critical value, is accelerating, and is flat. The acceleration implies the existence of cosmic dark energy that overcomes the gravitational self-attraction of matter and causes the expansion to speed up. This has once more drawn attention to the possible existence, at the present epoch, of a small positive cosmological term. Determination of the Hubble constant also seem to point in the same direction [1].

Albrecht and Magueijo [2] have investigated possible cosmological consequences of a time variation in the velocity of light in vacuum. In particular it offers new ways of resolving the problems of the standard Big-Bang cosmology, distinct from their resolutions in the context of the inflationary paradigm [3] or the pre-Big-Bang scenario of low-energy string theory [4. Moreover, in contrast to the case of the inflationary universe, varying $c$ may provide an explanation for the relative smallness of the cosmological constant today. An action formalism describing the speed of light as a minimally coupled scalar is given in Ref. [5]. We will follow this formalism. Several other theories including a variable speed of light have been proposed recently [6] [7] [8].

In refs. [2] and [5] it was considered a model containing a fluid whose equation of state obeys a linear baryotropic law with constant adiabatic index. The study presented in ref.[2] envisages a sudden fall in the speed of light, precipitated by a phase transition or some shift in the values of the fundamental constants, and explores the general consequences that might follow from a sufficiently large change. On the other hand in [5] it was proposed that the speed of light has a power-law dependence on the scale factor.

In this paper we investigate a varying speed of light scenario for a perfect fluid without restriction on the equation of state. We identify the attractor solutions and use them to perform the calculations. Thus we show that the cosmological field equations can be solved in general, defining the law of variation for $c(t)$. This allows us to give simple solutions to the flatness, coincidence, quasiflatness and horizon problems [9] [10] [11], even when the strong energy condition is satisfied. 


\section{The variable $c$ model}

In [2] it was argued that a time-variable $c$ should not introduce changes in the curvature of the space-time in the cosmological frame and that Einstein's equations must still hold. They have assumed that the universe is spatially homogeneous and isotropic, so that there are no spatial variations in $c$ or $G$. This leads to the requirement that the Lemaitre equations still retain their form with $c(t)$ and $G(t)$ varying. Thus the expansion scale factor obeys the equations

$$
\begin{aligned}
\frac{\dot{a}^{2}}{a^{2}} & =\frac{8 \pi G \rho}{3}-\frac{K c^{2}(t)}{a^{2}} \\
\ddot{a} & =-\frac{4 \pi G}{3}\left[\rho+\frac{3 p}{c^{2}(t)}\right] a
\end{aligned}
$$

where $p$ and $\rho$ are the density and pressure of the perfect fluid, and $K$ is the metric curvature parameter. This perfect fluid may comprise several components like clustered matter with energy density $\rho_{m}$ and pressure $p_{m}$, and a cosmological term that may be described as a perfect fluid with a stress satisfying $p_{\Lambda}=-\rho_{\Lambda} c^{2}$ where

$$
\rho_{\Lambda}=\frac{\Lambda c^{2}}{8 \pi G}
$$

From (11) and (8), we find the generalized conservation equation incorporating possible time variations in $c(t)$ and $G(t)$,

$$
\dot{\rho}+3 \frac{\dot{a}}{a}\left(\rho+\frac{p}{c^{2}}\right)=\frac{3 K c \dot{c}}{4 \pi G a^{2}}-\rho \frac{\dot{G}}{G} .
$$

The cosmological density parameter $\Omega$ is defined as the ratio of as the density

of the universe with the critical density $\rho_{c}=(3 / 8 \pi G) H^{2}$, where $H$ is the Hubble variable. The critical density defines the $K=0$ solution of Eq. (1). The fractional contributions to the right-hand side of Eq. (11), are given by $\Omega_{m} \equiv \rho_{m} / \rho_{c}, \Omega_{\Lambda} \equiv \rho_{\Lambda} / \rho_{c}=\Lambda c^{2} /\left(3 H^{2}\right)$, and $\Omega_{K} \equiv-K c^{2} /(a H)^{2}$, respectively. Thus, inserting

$$
\Omega \equiv \frac{\rho}{\rho_{c}}=\frac{8 \pi G \rho}{3 H^{2}}
$$

in (1), we have 


$$
\frac{\Omega}{\Omega-1}=\frac{8 \pi G \rho a^{2}}{3 K c^{2}(t)} .
$$

Differentiating it, and using the conservation equation (州) we find the dynamical equation for the cosmological density parameter

$$
\dot{\Omega}=(\Omega-1)\left[\left(1+\frac{3 p}{\rho c^{2}}\right) \Omega H+\frac{2 \dot{c}}{c}\right] \equiv f(\Omega)
$$

It is important to obtain exact solutions of Eq. (7) in order to evaluate the effects of a perfect fluid, varying $G$ and $c$ on the expansion dynamics. In particular, as we shall see bellow, stable constant solutions of this equation are relevant to solve several cosmological conundrums like the flatness, coincidence, quasi-flatness, and horizon problems. Eq. (7) has three constant solutions: $\Omega=0$, when $\dot{c}=0$, that corresponds to the Milne universe, $\Omega=1$ that represents the flat universe and $\Omega=\Omega_{0}$ that arises when the squared bracket in (7) vanishes. To show that these solutions are dynamical attractors we will use the fact that a constant solution $f\left(\Omega_{s}\right)=0$ of (7) is asymptotically stable provided $f^{\prime}\left(\Omega_{s}\right)<0$.

\section{The Flatness Problem}

The combined measurements of the cosmic microwave background temperature fluctuations and the distribution of galaxies on large scales began to suggest that the universe may be flat [12]. Within standard General Relativity, where $\dot{G} \equiv 0 \equiv \dot{c}$, the conservation equation (四) gives

$$
\rho=\rho_{0} \exp \left[-3 \int d t\left(1+\frac{p}{\rho c^{2}}\right) H\right]
$$

Hence the curvature term will dominate the matter density term at large $a$ whenever the matter stress obeys the strong energy condition (SEC) $\rho+$ $3 p / c^{2} \geq 0$. Equivalently, Eq. (7) shows the solution $\Omega=1$ is unstable so that extreme fine tuning of the conditions at the early universe seems to be required to accommodate the observations. This is called the flatness problem [9]. The solution postulated by inflation requires a sufficiently long period of evolution in the early universe during which the expansion was dominated by a gravitationally repulsive stress that violated SEC. In this 
way the evolution would have been driven very close to $\Omega=1$. Also Eq. (2) implies a period with superluminal expansion as $\ddot{a}>0$.

From Eq. (17) we see that a decreasing speed of light $(\dot{c} / c<0)$ would also drive $\Omega$ to 1 . In fact, when the speed of light changes with $\dot{c} / c<$ $-\left(1+3 p / \rho c^{2}\right) H / 2$, the solution $\Omega=1$ is asymptotically stable. Thus in a varying speed of light (VSL) scenario the flatness problem can be solved even when SEC is satisfied. No fine-tuning of the initial conditions is needed in this scenario and no further specification of the equation of state is required.

\section{The Coincidence Problem}

In the last years there has been a renewed interest in the possibility that a positive cosmological constant may dominate the total energy density in the universe. Interest in the cosmological constant stems from several directions. Dynamical estimates of the amount of clustered matter yield a conservative

upper limit $\Omega_{m} \lesssim 0.3$, and recent observations of Type 1a supernovae indicate an accelerating universe. Combining them with the observations suggesting $\Omega_{K} \simeq 0$ we conclude that $\Omega_{m}+\Omega_{\Lambda} \simeq 1$, where the density ratio parameter $\epsilon=\rho_{m} / \rho_{\Lambda}=\Omega_{m} / \Omega_{\Lambda}$ has the current value $\epsilon_{0} \simeq 3 / 7$ [13].

The puzzle with the cosmological term is explaining why $\rho_{\Lambda}$ and the matter energy density $\rho_{m}$ should be comparable today. Throughout the history of the universe the two densities decrease at different rates and so it appears that the conditions in the early universe have to be set very carefully in order for the energy densities to be comparable today. We refer to this issue of initial conditions as the coincidence problem [10.

From these observational evidences, it is natural to take a step beyond Einstein's original hypothesis and consider that the $\Lambda$-term is not a constant, but rather, describes a new dynamical degree of freedom. Neither observational data, nor inflationary considerations tell us that a cosmological term is constant. Between different mechanisms explored in the literature to solve this problem the VSL theory provides us a simple way to obtain a decaying effective cosmological term $\Lambda c(t)^{2}$. Following [2] we adopt this model to find a stable solution of the Einstein equations with constant $\epsilon$.

Integrating (4) for $\Omega=1$ we get

$$
\rho(t)=\frac{\rho_{0}}{G} \exp \left[-3 \int d t\left(1+\frac{p}{\rho c^{2}}\right) H\right]
$$


where $\rho=\rho_{m}+\rho_{\Lambda}$. Hence, using ([3) we find that $\epsilon$ has the constant solution

$$
\epsilon_{0}=\frac{8 \pi \rho_{0}}{\Lambda c_{0}^{2}}-1
$$

provided that the speed of light decreases as

$$
c(t)=c_{0} \exp \left[-\frac{3}{2} \int d t\left(1+\frac{p}{\rho c^{2}}\right) H\right]
$$

where $c_{0}$ is a positive constant (we assume the dominant energy condition throughout). From (11) and (9) we have

$$
\rho=\frac{\rho_{0} c^{2}}{c_{0} G}
$$

This solution is an attractor as in the neighbourhood of $\Omega=1$ we get $\dot{\Omega} \simeq$ $-2 H(\Omega-1)$. In this way we have solved the coincidence problem in the framework of the VSL scenario as $\epsilon$ approaches the attractor solution (10) without a fine-tuning of the initial conditions and without restriction on the equation of state. In this asymptotic regime, assuming cold dark matter and using (11), we find that $c \simeq a^{-3 \epsilon_{0} /\left[2\left(1+\epsilon_{0}\right)\right]}$.

\section{Quasiflatness problem}

In general it is accepted that our universe has a low-mass-density $\left(\Omega_{m}<\right.$ 1 ). The determination of the universe's mass density is currently the beststudied cosmological parameter and its low value is indicated by a number of independent methods for the study of clusters of galaxies. They include the mass-to-light ratio, the baryon fraction, the cluster abundance and the mass power spectrum [13]. Thus, if the energy density of our universe were dominated by clustered matter we would find a problem related to the flatness problem: a low $\Omega$ universe with $\Omega \simeq O(1)$ also requires extreme fine tuning of initial conditions. This is the quasi-flatness problem and we shall see that it also has solution within the VSL framework.

The constraint associated with the constant solution $\Omega_{0}$ determines a first order differential equation for $c(t)$ whose general solution becomes

$$
c(t)=c_{0} a^{\Omega_{0}} \exp \left[-\frac{3 \Omega_{0}}{2} \int d t\left(1+\frac{p}{\rho c^{2}}\right) H\right]
$$


We note that this expression leads to a decreasing speed of light provided that SEC holds. We can solve (田) using (13)

$$
\rho(t)=\rho_{0} \frac{a^{2\left(\Omega_{0}-1\right)}}{G} \exp \left[-3 \Omega_{0} \int d t\left(1+\frac{p}{\rho c^{2}}\right) H\right]
$$

hence we find

$$
\rho=\frac{\rho_{0}}{c_{0}^{2}} \frac{c^{2}}{a^{2} G}
$$

Using (1 1 ), (可) and (15), we get this a relationship between the cosmological density parameter and the integration constants

$$
\Omega_{0}=\frac{1}{1-\frac{3 K c_{0}^{2}}{8 \pi \rho_{0}}}
$$

Integrating (11) for the energy density (15), we express the scale factor as

$$
a(t)=\sqrt{\frac{K}{\Omega_{0}-1}} \int c(t) d t
$$

Using (13), in a neighbourhood of the solution (16), Eq. ([7) becomes

$$
\dot{\Omega} \simeq\left(\Omega_{0}-1\right)\left(1+3 \frac{p}{\rho c^{2}}\right) H\left(\Omega-\Omega_{0}\right)
$$

Hence the solution $\Omega_{0}<1$ is an attractor provided SEC holds, solving the quasi-flatness problem for any perfect fluid that satisfies this condition.

In the particular case that we choose the linear baryotropic equation of state $p=(\gamma-1) \rho c^{2}(t)$ with constant adiabatic index $\gamma$, we obtain from (13)

$$
\begin{gathered}
c(t)=c_{0} a^{n} \\
\Omega_{0}=\frac{2 n}{2-3 \gamma}
\end{gathered}
$$

In this case SEC holds when $\gamma>2 / 3$ that means $n<0$ when $\Omega_{0}>0$. From (17) the scale factor is

$$
a(t) \propto \Delta t^{\frac{1}{1-n}}
$$

In this way our exact solution is the late time limit of the solution found in 11. 


\section{The Horizon Problem}

One of the most puzzling features of the Standard Big Bang model is the presence of cosmological horizons. At any given time any observer can only see a finite region of the Universe. Since the horizon size increases with time we can now observe many regions in our past light cone which are causally disconnected. The fact that these regions have the same properties is puzzling as they have not been in physical contact [3] 9]. This would have required an extraordinary fine-tuning of the initial conditions in the early Universe. Usually this problem is solved in the inflationary scenario again appealing to sufficiently long period violating SEC. We will show that a VSL scenario may also solve this problem without its violation.

The observed Universe was already smooth at the nucleosynthesis era $(\simeq 1 \mathrm{~s})$. Hence the horizon problem has to be solved at an earlier time, $t_{1}$ say. The size of the connected region at time $t_{1}$ with events at an initial time $t_{0}$ is proportional to the integral

$$
\int_{t_{0}}^{t_{1}} d t^{\prime} \frac{c\left(t^{\prime}\right)}{a\left(t^{\prime}\right)}
$$

and the particle horizon corresponds to the limit $t_{0} \rightarrow 0$. When the curvature becomes negligible the scale factor for the scenario solving the coincidence problem is given by

$$
a(t)=a_{0} \exp \left[\sqrt{\frac{8 \pi \rho_{0}}{3}} \int d t \frac{c}{c_{0}}\right]
$$

In this case the integral (22) diverges in the limit $t_{0} \rightarrow 0$. The same occurs when this integral is evaluated using the expressions for the speed of light (13) and scale factor (17) of the scenario solving the quasiflatness problem. Hence in the VSL cosmology the size of the connected region at $t_{1}$ can be made arbitrary larger than the size of our past light cone at $t_{1}$ by choosing the initial time $t_{0}$ small enough. This solves the horizon problem without violation of SEC.

\section{Discussion}

In this letter we have considered a varying- $c$ theory recently proposed by Albrecht and Magueijo [2] as a new way of solving the flatness, coincidence, 
quasiflatness and horizon problems for any perfect fluid.

We have found the constant solutions for the cosmological density parameter of the Einstein equations and the conditions that determine when these solutions are attractors for an expanding universe. In this way we have solved several problems of the Standard Big Bang cosmology without requiring a stage when the strong energy condition is violated. No specific form for the equation of state of the perfect fluid has been imposed, and in this way a wide range of matter sources can be accommodated within our model. Also we have proposed a better way to understand a small but nonzero cosmological constant, as indicated by a number of recent observational studies.

It is interesting to mention that a time dependent $G$ cannot solve the flatness, coincidence or quasiflatness problems as it does not appear in Eq. (7). We have not touched in this letter the issue of entropy production within the VSL scenario. This will be the subject of a future paper.

\section{Acknowledgements}

This research has been supported by the University of Buenos Aires under project TX-93. We are grateful to the University of the Basque Country for partial support under project UPV 172.310-G02/99.

\section{References}

[1] W. Freedman et al., Nature 371 (1994) 757.

[2] A. Albrecht and J. Magueijo, Phys. Rev. D 59 (1999) 043516.

[3] A.H. Guth, Phys. Rev. D 23 (1981) 347.

[4] G. Veneziano, Phys. Lett. B 406 (1997) 297.

[5] J.D. Barrow Phys. Rev. D 59 (1999) 043515.

[6] M.A. Clayton and J.W. Moffat, PLB 460 (1999) 263, Report grqc/9910112.

[7] J. W. Moffat, Report astro-ph/9811390.

[8] I. T. Drumond, Report gr-qc/9908058. 
[9] R.H. Dicke and P.J.E. Peebles, in: General Relativity: An Einstein Centenary Survey, eds. S.W. Hawking and W. Israel (Cambridge U. Press, 1979).

[10] P. Steinhardt, in: Critical Problems in Physics, eds. V.L. Fitch and D.R. Marlow (Princeton U. Press, 1997).

[11] J.D. Barrow and J. Magueijo, Phys. Lett. B 447 (1999) 246.

[12] J. P. Ostriker and P. J. Steinhardt, Nature 377 (1995) 600.

[13] N.A. Bahcall, J. P. Ostriker, S. Perlmutter and P.J. Steinhardt, Science 284 (1999) 1481. 